\title{
A Concealed Car Extraction Method Based on Full-Waveform LiDAR Data
}

\author{
Chuanrong Li, ${ }^{1}$ Mei Zhou, ${ }^{1}$ Menghua Liu, ${ }^{1}$ Lian Ma, ${ }^{1}$ and Jinhu Wang ${ }^{2}$ \\ ${ }^{1}$ Key Laboratory of Quantitative Remote Sensing Information Technology, Academy of Optoelectronics, \\ Chinese Academy of Sciences, Beijing 100094, China \\ ${ }^{2}$ Department of Geoscience and Remote Sensing, Delft University of Technology, 2628CN Delft, Netherlands
}

Correspondence should be addressed to Mei Zhou; zhoumei@aoe.ac.cn

Received 9 February 2016; Revised 3 June 2016; Accepted 21 July 2016

Academic Editor: Sander O. Elberink

Copyright (C) 2016 Chuanrong Li et al. This is an open access article distributed under the Creative Commons Attribution License, which permits unrestricted use, distribution, and reproduction in any medium, provided the original work is properly cited.

\begin{abstract}
Concealed cars extraction from point clouds data acquired by airborne laser scanning has gained its popularity in recent years. However, due to the occlusion effect, the number of laser points for concealed cars under trees is not enough. Thus, the concealed cars extraction is difficult and unreliable. In this paper, 3D point cloud segmentation and classification approach based on fullwaveform LiDAR was presented. This approach first employed the autocorrelation $G$ coefficient and the echo ratio to determine concealed cars areas. Then the points in the concealed cars areas were segmented with regard to elevation distribution of concealed cars. Based on the previous steps, a strategy integrating backscattered waveform features and the view histogram descriptor was developed to train sample data of concealed cars and generate the feature pattern. Finally concealed cars were classified by pattern matching. The approach was validated by full-waveform LiDAR data and experimental results demonstrated that the presented approach can extract concealed cars with accuracy more than $78.6 \%$ in the experiment areas.
\end{abstract}

\section{Introduction}

Automatic object extraction becomes a more and more active research topic over the last decades [1]. Some researchers attempt to automatically identify and extract objects from traditional remote images. However successful scenarios are limited. Since the radiometric properties of objects are complicated and dynamic, algorithms that work well with one set of 3D objects are not suitable for other data sets [2]. Moreover, information of targets under vegetation coverage cannot be included in imagery. Thus, extraction of concealed targets cannot be realized based only on images. LiDAR (Light Detection and Ranging) data has unique properties for extraction of 3D objects. Because laser pulse can penetrate vegetation to obtain targets information, LiDAR has irreplaceable superiorities compared to traditional remote images in extraction of concealed targets under vegetation coverage [3]. The most common concealed targets under vegetation coverage are concealed cars. Concealed cars extraction from airborne LiDAR data can be potentially applied to many fields, such as military surveillance, homeland security, global warming, disaster rescue, emergency road service, and criminal searching [4].

Some investigations have been conducted on cars extraction using 3D LiDAR point clouds. Yao et al. [5] presented a method to extract individual car from common LiDAR data in urban areas. Firstly, ground level separation was used to exclude the irrelevant objects and provide the "Region of Interest." Then the marker-controlled watershed transformation assisted by morphological reconstruction was performed on the gridded and filled raster of ground level points to delineate the single cars. The evaluation of experimental results showed the high potential of airborne LiDAR in outlining single cars in urban areas, which allowed accurate 3D point retrieval of single car. To reliably extract car targets from LiDAR data, Sun et al. [6] proposed a pixel and object oriented method for car extraction based on elevation and intensity data after filtering. The experimental results demonstrated the feasibility of the method. Borcs and Benedek [7] proposed a novel two-level MPP (Marked Point Process) model for the extraction of cars and traffic segments in airborne laser point cloud data. The efficiency of 
the approach was evaluated with LiDAR data. However, these methods were applied to extract cars on bare ground. Though all bare ground point extraction algorithms performed well on LiDAR point clouds from smooth rural landscapes, they produced errors in rough terrain with vegetation canopy. Chang et al. [8] built a system to detect cars underneath canopy in forested terrain from LiDAR point clouds. They developed an automatic canopy removal algorithm and a novel bare-earth extraction algorithm to reveal the LiDAR points underneath forest canopy and filter ground points, respectively. All obscure cars underneath tree canopy were revealed as demonstrated. It showed that the state-of-theart airborne LiDAR system can provide valuable data which can effectively support the occluded car extraction in forest terrain. However, only the elevation and intensity features were used to extract the concealed cars in the method.

Since the traditional LiDAR records only several returned echoes and acquires limited information of objects, only range and intensity information can be used to extract the concealed cars. Due to the reflection from canopy, the intensity of points from concealed cars under vegetation is not distinguishable from that of their neighborhood points [9]. Thus, it is difficult to extract the concealed cars under vegetation using traditional discrete LiDAR point cloud data. Unlike traditional discrete LiDAR, waveform features characterizing the inherent attributes of targets can be extracted by processing full-waveform LiDAR data [10, 11]. The features, including number of peaks, Full Width at Half Maximum (FWHM), energy of the returned waveform, and backscattering cross section, can be extracted for targets classification [12-15]. Moreover, compared to the points directly obtained by LiDAR systems, point density can be improved to a certain extent by employing waveform features [11]. In this way, the vertical structure information of targets can be obtained more accurately. Therefore, the performance of target extraction can be improved by using the point cloud data affiliated with waveform features. In this study, the full waveform data was first applied to the concealed target extraction which was in line with the current development trend of the target extraction.

Influenced by many factors, such as environment and characteristics of concealed targets, the number of backscattered waveform from concealed targets is less than that of uncovered targets, which results in inadequate waveform features of concealed targets. Therefore, VFH (Viewpoint Feature Histogram) descriptors combined with waveform features were used for concealed cars extraction. VFH [16] is a global descriptor for $3 \mathrm{D}$ point cloud data that encodes geometry and viewpoint. Alhamzi et al. [17] have used stateof-the-art 3D descriptors to recognize the objects and evaluated their relative performance. Based on the experimental results, the VFH had the best performance to recognize the objects among other global descriptors. Ceron and Prieto [18] evaluated different combination of three descriptors that are suitable for object recognition and classification: Spin Images, VFH, and NARF (Normal Aligned Radial Feature). The two experiments showed that the VFH outperformed other 3D Shape Descriptors when used alone. Because VFH has high recognition performance and fast computational properties, it has been widely used for objects recognition and classification [16, 19-21]. In this paper, the VFH of the point cloud data affiliated with waveform features was calculated and employed for concealed cars extraction. The approach was verified using full-waveform LiDAR data. The results showed that concealed cars extraction can be realized using the approach.

\section{Theoretical Background}

The objective of this study is to extract concealed cars based on full-waveform LiDAR data. Considering the waveform characteristics of the concealed cars, spatial statistical analysis method was introduced for 3D point cloud segmentation. 3D geometry features and waveform features were combined for points classification.

2.1. Spatial Statistical Analysis. Due to the influence of terrain and environment, the spatial distribution of the waveform echo index from different targets varies. The waveform echo index denotes the position of the echo in the backscattered waveform [22]. The obtainment of such waveform echo index requires a preprocessing step. By waveform decomposition, a number of echoes can be extracted, and then waveform echo index can be obtained. The aim of this step is to extract all (or most of the) relevant peaks in order to generate a dense 3D point cloud [11].

In this paper, the concealed cars refer to the cars under the coverage of vegetation. In general, the echo index value of vegetation area is bigger than that of ground area. Thus spatial statistical analysis of echo index can be used to determine the vegetation areas. The spatial autocorrelation [23], which is one of the most important concepts in spatial statistics, is used to describe the correlation for echo index in different locations. The spatial autocorrelation has two indices, named global index and local index, respectively. The global index [24] is used to detect the spatial autocorrelation of the entire study area. A single value is used to outline the degree of spatial autocorrelation for the entire study area. The local index [25] assesses the extent to which observations of similar and nonsimilar values are clustered for each individual location. Currently the widely used global and local spatial autocorrelation indices are Moran index and $G$ coefficient $[23,26,27]$. Compared with the global Moran index which can only find the similarity value (positive correlation) or nonsimilarity values (negative correlation) in spatial aggregation model, the global $G$ coefficient is capable of detecting whether the unit belongs to high value aggregation or low value aggregation of echo index in spatial distribution pattern [23]. The detecting ability for spatial clustering of local Moran index and local $G$ coefficient also has significant differences. The Moran index can roughly detect the cluster center but is unable to discriminate whether a pattern is dominated by high values or low values. But $G$ coefficient can accurately detect the cluster [26]. The vegetation areas are isolated regions; thus the local $G$ coefficient is used for spatial statistical analysis on the waveform echo index of concealed cars areas in this work. 
The local $G$ coefficient [24] measures local concentration by calculating the ratio of the sums of attribute values between local neighborhood and the entire region. The calculation formula of local $G$ coefficient is [26]

$$
G_{i}(d)=\frac{\sum_{j=1}^{n} W_{i j}(d) x_{j}}{\sum_{j=1}^{n} x_{j}}, \quad j \text { not equal to } i,
$$

where $x_{j}$ denotes the echo index value of the $j$ th point; $\left\{W_{i j}\right\}$ is a symmetric one/zero spatial weight matrix with ones for all links defined as being within distance $d$ of a given point $i$; all other links are zero including the link of point $i$ to itself. The numerator is the sum of all $x_{j}$ within $d$ of $i$ but not including $x_{i}$. The denominator is the sum of all $x_{j}$ within the entire region under investigation not including $x_{i}$. The standardization of local $G$ coefficient is [26]

$$
Z\left(G_{i}\right)=\frac{G_{i}-E\left(G_{i}\right)}{\sqrt{\operatorname{VAR}\left(G_{i}\right)}},
$$

where $E\left(G_{i}\right)$ is the value of expectation and $\operatorname{VAR}\left(G_{i}\right)$ is the variance. $A$ large positive $Z\left(G_{i}\right)$ implies that points with high echo index are within $d$ of point $i$. A large negative $Z\left(G_{i}\right)$ means that low echo index points are within $d$ of point $i$ $[24,26]$.

2.2. Description of $3 D$ Point Features. As mentioned, the number of backscattered waveforms from concealed targets is less than that of uncovered targets. Less waveform features of concealed targets are obtained. Especially for concealed cars, even less waveform features can be acquired due to the small size. In order to more effectively extract the concealed cars, not only the waveform features but also the $3 \mathrm{D}$ point features were utilized. The Point Feature Histograms (PFH) descriptor $[28,29]$ is a $3 \mathrm{D}$ feature descriptor, the purpose of which is to encode the local neighborhood's geometrical properties by generalizing the mean curvature at a point $P_{q}$ using a multidimensional histogram of values. In its most basic form, the computation of PFH at a point $P_{q}$ relies on the presence of $3 \mathrm{D}$ coordinates and estimated surface normals [30]. The computation steps are as follows: (i) For each point $P_{q}$, all of $P_{q}$ 's neighbors enclosed in the sphere with a given radius $r$ are selected ( $k$-neighborhood). (ii) For every pair of points $p_{i}$ and $p_{j}(i \neq j)$ in the $k$-neighborhood of $P_{q}$ and their estimated normals $n_{i}$ and $n_{j}$, a Darboux $u n v$ frame $\left(u=n_{i}, v=\left(p_{j}-p_{i}\right) \times u, w=u \times v\right)$ is defined and the angular variations of $n_{i}$ and $n_{j}$ are computed as follows:

$$
\begin{aligned}
& \alpha=v \cdot n_{j}, \\
& \phi=\frac{\left(u \cdot\left(p_{j}-p_{i}\right)\right)}{\left\|\left(p_{j}-p_{i}\right)\right\|}, \\
& \theta=\arctan \left(w \cdot n_{j}, u \cdot n_{j}\right) .
\end{aligned}
$$

These three features are measurements of the angles between the points' normals and the distance vector between

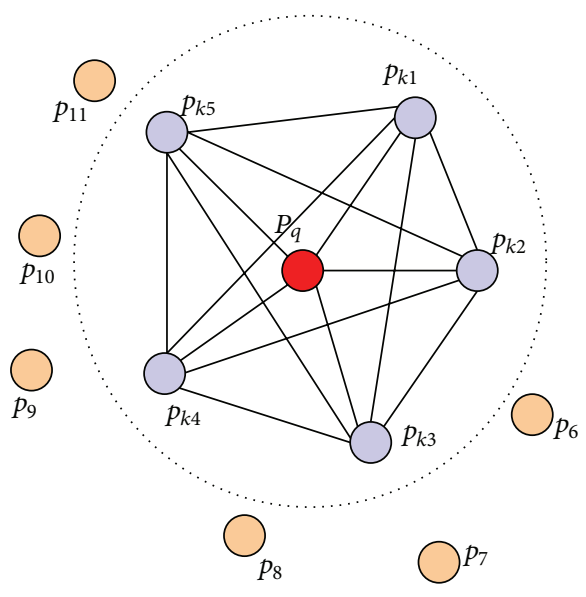

FIGURE 1: The influence region diagram for a PFH calculation. The query point $P_{q}$ (red) and its $k$-neighborhood (blue) are fully interconnected in a mesh.

them. The histogram collects these three angles between every pair of normals on a surface patch [28].

The influence region in the calculation of $\mathrm{PFH}$ for the query point $P_{q}$ is shown in Figure 1 [30]. The query point $P_{q}$ is marked with red in the middle position of a circle (sphere in $3 \mathrm{D}$ ) whose radius is $r$, and all its $k$-neighborhood (points with distances smaller than the radius $r$ ) is fully interconnected in a mesh [30]. When all possible pairs of points are considered, the computational complexity for $k$-neighborhood is $O\left(k^{2}\right)$. Thus the theoretical computational complexity of the PFH for a given point cloud with $\mathrm{n}$ points is $O\left(n \cdot k^{2}\right)$ [29], where $k$ is the number of neighbors for each query point in a point cloud.

In order to simplify the calculation of PFH, the Fast Point Feature Histogram (FPFH) is introduced. The calculation complexity of FPFH is reduced to $O(n k)$, but it still preserves most of the discriminative ability of PFH [16]. The specific calculation steps for FPFH are as follows:

(i) For each query point $P_{q}$, the relationships (see (3)) between $P_{q}$ and its neighbors are only calculated. The first step is called Simple Point Feature Histograms (SPFH).

(ii) The $k$ neighbors for each point are redetermined, and the neighboring SPFH values are used to weight the final histogram of $P_{q}$ (called FPFH); the specific formula is

$\operatorname{FPFH}\left(P_{q}\right)=\operatorname{SPFH}\left(P_{q}\right)+\frac{1}{k} \sum_{i=1}^{k} \frac{1}{w_{k}} \cdot \operatorname{SPFH}\left(P_{k}\right)$,

where the weight $w_{k}$ means the distance between the query point $P_{q}$ and its neighbor point $P_{k}$ in a given metric space.

Viewpoint Feature Histograms (VFH) descriptor is derived from FPFH and is mainly used in 3D object recognition and classification [16]. The VFH adds a viewpoint variance while retaining its invariance to scale, as shown 


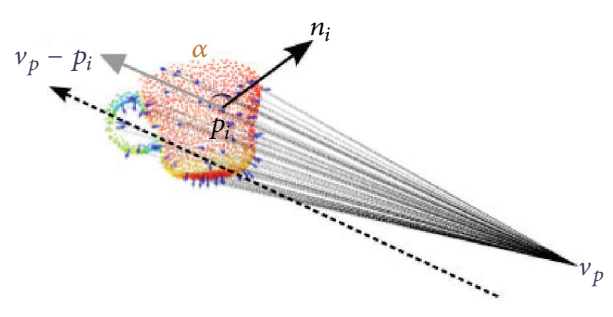

FIGURE 2: The schematic diagram of viewpoint direction component calculation between the view direction and the normal.

in Figure 2 [20]. Thus VFH descriptor consists of two parts: a viewpoint direction component and surface shape component comprised of an extended FPFH [16]. To compute the first part, the centroid of the object is found, which is the point that results from averaging the three coordinates of all points. Next, the vector between the viewpoint and the centroid is computed and normalized. Finally, for all points in the cluster, the angles between this vector and their normals are calculated. The result is represented by a histogram. Note that it does not mean the view angle to each normal as this would not be scale invariant. Instead, it means the angle in the central viewpoint direction translated to each normal. The second part is computed like the FPFH. The resulting four histograms (one for the viewpoint component and three for the extended FPFH component) are concatenated to build the final VFH descriptor [17].

2.3. Waveform Feature Extraction. By decomposing the returned waveforms, waveform features, which are used for concealed cars 3D point cloud segmentation and classification, can be extracted. The extracted waveform features in this paper include distance, intensity, pulse width, backscattering cross section, and echo index. The distance feature indicates the distance from laser transmitter to the target which is determined by estimating the waveform echo position [11]. Ideally, the peak position is considered as component position to calculate the distance. Intensity is not yet a clearly defined term. The echo amplitude is most commonly referred to as intensity [31]. The return intensity is a function related to the reflective capability of the targets. The waveform width denotes the depth extension of waveform in the laser incident direction, which is closely related to the geometry of targets, terrain slope, and targets material [10]. The backscattering cross section delineates the backscattering ability of the targets and is a comprehensive indicator of distance, intensity, and waveform width. It is not a practical geometric area, but the equivalent area that scatters laser pulse back to receiver after the transmitted laser pulse illuminates a target. It can be obtained by the laser energy transmission equation after calibration [32]. $G$ coefficient and echo ratio (ER) can be calculated based on echo index. $G$ coefficient was introduced in Section 2.1. Echo ratio refers to the ratio between the total number of points in a sphere with the radius of $r$ and the total number of points in a cylinder with the radius of $r$ [33]. The most suitable value of $r$ is two times the average distance between two points.

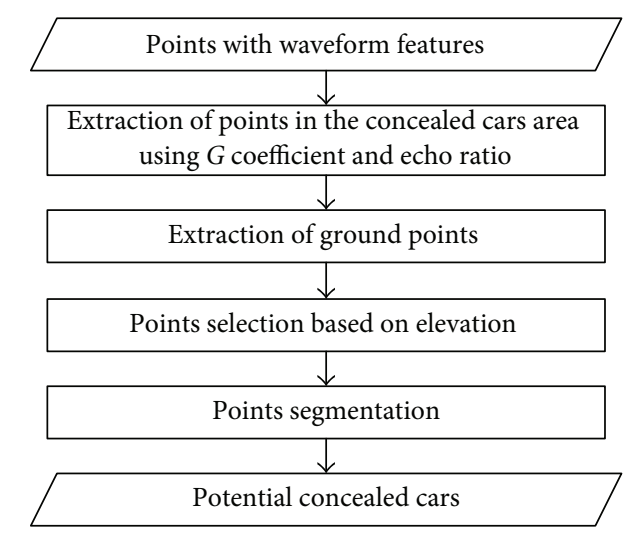

FIGURE 3: Flowchart of 3D point cloud segmentation.

Some factors, such as angle of incidence, atmospheric correction, range, and surface characteristics, have influence on the waveform features. Therefore, these features can hardly be used without radiometric calibration [34]. To weaken such influence and further improve the effectiveness of waveform features for concealed cars extraction, this paper has made a comprehensive correction on the extracted waveform features. The detailed methodology was introduced in [35].

\section{Concealed Cars Extraction Approach}

3.1. 3D Point Cloud Segmentation. Using the characteristics of high value aggregation of the waveform echo index for the concealed cars areas, spatial autocorrelation $G$ coefficient and the echo ratio were introduced to determine the concealed cars area. Considering the differences of waveform features between ground and nonground points, ground information of concealed cars area was extracted firstly. Then the points within the elevation range of concealed cars were segmented. The workflow is illustrated in Figure 3.

3D point cloud segmentation consists of three steps:

(1) Waveform features were first extracted through the decomposition of backscattered waveforms. Next $G$ coefficient and echo ratio were calculated. Then $G$ coefficient and echo ratio were combined to classify points so that the points in the concealed cars area can be extracted.

(2) Ground point information in the concealed cars area was extracted using a split-and-merge segmentation algorithm based on an octree structure proposed by Wang and Tseng in [36]. Consecutively, the points within the elevation range of concealed cars were extracted. Considering the characteristics of the cars, the elevation range was set to $1 \mathrm{~m}-4 \mathrm{~m}$ from the ground. This reduced the influence of uninterested points on 3D point cloud segmentation.

(3) The features for each extracted 3D point (one echo) were combined to form a feature vector ( $X, Y, Z, A, W, \mathrm{CS}, G, \mathrm{ER})$, where $X, Y$, and $Z$ were $3 \mathrm{D}$ coordinates of the points and $A, W, \mathrm{CS}, G$, and ER 
were intensity, waveform width, backscattering cross section, $G$ coefficient, and echo ratio, respectively. The distance between the feature vectors of different points was calculated. Then KD-tree [37] which was an index structure for storing a finite set of points from a $k$-dimensional space was used for nearest neighbor search in accordance with the distance. For a query point $p$, radius searching was performed. All its neighbors that were within radius $d$ were put into a cluster. The same procedure was conducted for the searched neighborhood points until there was no new point that could be put in the cluster, or the number of points in the cluster reached threshold value. In this paper, the distance $\mathrm{d}$ was set to 1.5 times the average distance between two points of the experiment data. The minimum number of points for a cluster was set to 40 according to the actual experiment data. In this way, the points that remained after the second step could be clustered.

3.2. Classification. Due to the small size of concealed cars, only a few points on concealed cars can be captured. To classify concealed cars more effectively, more point cloud features reflecting the attributes of cars were required. In this paper, waveform features (distance, intensity, waveform width, and backscattering cross section) and VFH descriptors were combined for classification of point clusters. First, sample data was used for training and KD-tree was built. Then the nearest neighbor searching for the input cluster data was implemented based on the built KD-tree. The distances between the objects in the sample data and input cluster data were obtained and sorted. Then the matching result was determined according to the minimum distance. If the minimum distance was less than the threshold, then the input cluster data was classified as concealed car. The whole process consists of training stage and classification stage.

The flowchart of the training stage is given in Figure 4. The detailed steps are as follows: (1) Collect the full waveform data of different types of cars. Mean, variance and maximum and minimum values of waveform features and VFH descriptors were calculated. The points of concealed cars containing the waveform features and VFH were used as sample data for training. (2) The features of each sample were stored as point cloud feature files and added to a sample library. (3) Based on the traversal of point cloud feature files in the sample library, a KD-tree index was built and saved for subsequent use.

The flowchart of classification stage is shown in Figure 5. This procedure consists of three steps: (1) The point cloud feature files generated in training stage were loaded, and then the KD-tree index was rebuilt. (2) The clusters in Section 3.1 were set as target sets for classification. Mean, variance, and maximum and minimum values of waveform features and VFH descriptors were calculated. (3) Nearest neighbor searching was conducted using the waveform features and VFH descriptors based on KD-tree built in step (1). The sample data were searched, and feature distances between samples and target sets were calculated. (4) One determined whether the minimum distance of features between the

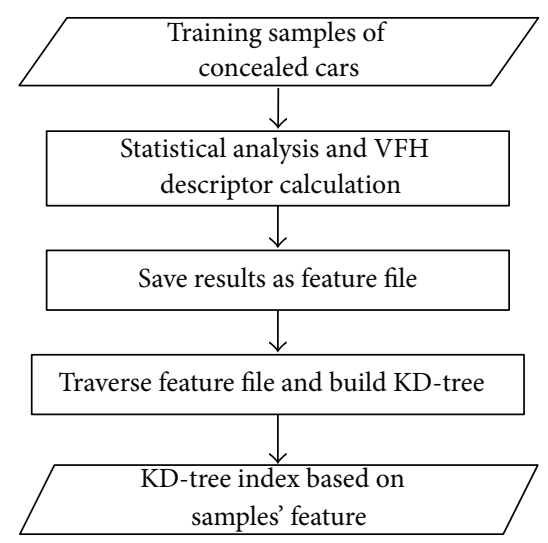

FIGURE 4: Flowchart of concealed car samples training.

sample and target set was less than a preset threshold, which was determined by the variance of the features. If so, then the target set was classified as a concealed car. Otherwise the target set was classified as other targets, such as the dense thickets or bare cars. Finally the classification results were output.

\section{Experiment and Results Analysis}

4.1. Data Description. The data used in this paper was acquired by airborne small-footprint full-waveform LiDAR system LMS-Q560 from Riegl. The flight height is 700 meters, the wavelength of the laser is $1550 \mathrm{~nm}$, the divergence angle of laser beam is $0.5 \mathrm{mrad}$, and the range measurement accuracy is 0.15 meters. The point cloud density is 4 points $/ \mathrm{m}^{2}$. Figure 6 shows an example of concealed cars and the corresponding point cloud. Figure $6(\mathrm{a})$ is a photograph of cars under vegetation and Figure 6(b) is a side view of the original point cloud data of the same area. Three different experiment areas with different vegetation coverage and targets distribution, which were named as experiment area I, experiment area II, and experiment area III in Miyun in Beijing, were selected to test the presented concealed cars extraction approach. The CCD image and original point cloud data which is colorized by elevation are, respectively, shown in Figures 7(a), 7(b), 8(a), 8(b), 9(a), and 9(b). In order to show the position of concealed cars clearly, the point cloud data of concealed cars are visualized using the highlight red color, as shown in Figures $7(\mathrm{c}), 8(\mathrm{c})$, and $9(\mathrm{c})$. There are 15,15 , and 20 concealed cars in experiment areas I, II, and III, respectively.

4.2. Experiment. The results of concealed cars areas are illustrated in Figures 10, 11, and 12. It shows that the $G$ coefficient can be used to extract the big area of trees and the echo ratio can be used to extract the boundary of the trees. Then points within the elevation range of concealed cars in the concealed cars areas were segmented, as illustrated in Figures 10(c), 11(c), and 12(c). A total number of 96, 28, and 82 potential concealed cars were segmented, respectively, for experiment areas I, II, and III. The concealed cars were all 


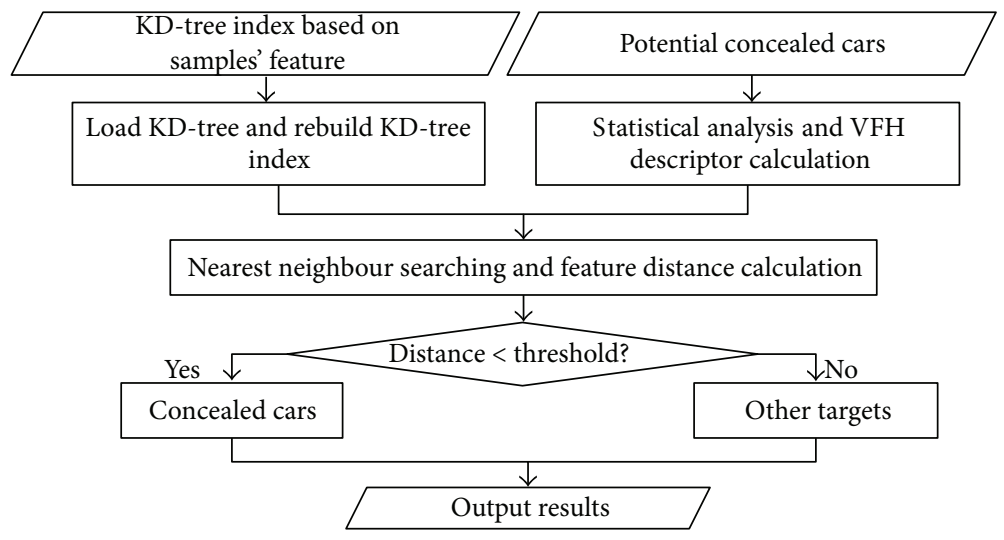

FIGURE 5: Flowchart of concealed cars classification.

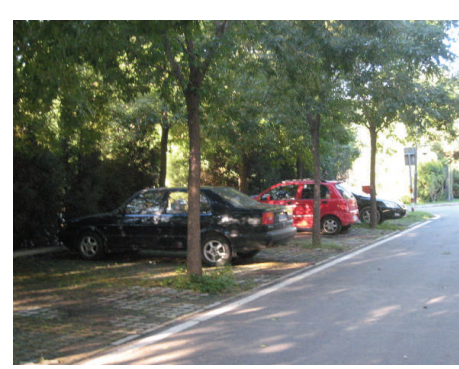

(a) Photograph

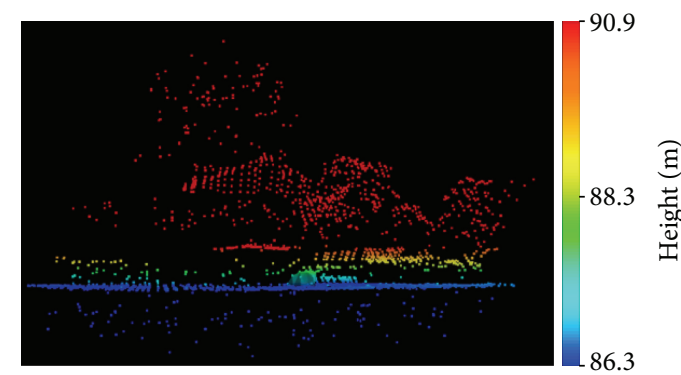

(b) The side view of original point cloud data

FIGURE 6: Concealed cars under trees.

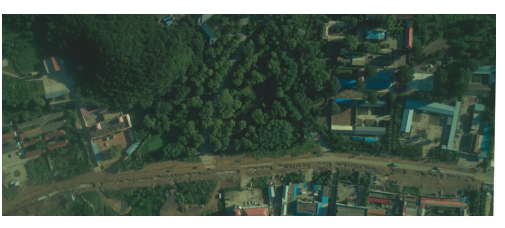

(a) CCD image

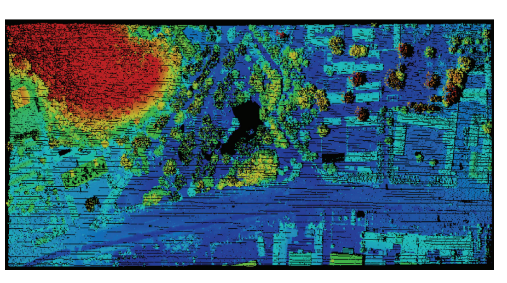

(b) Original point cloud data

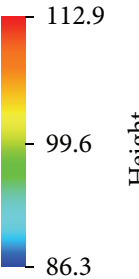

cars

FIGURE 7: The CCD image, original point cloud data, and position of concealed target cars in experiment area I.

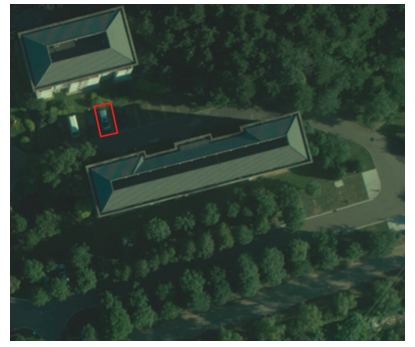

(a) CCD image

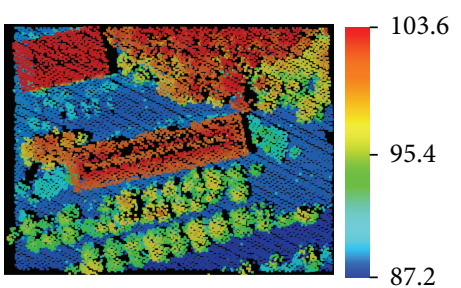

(b) Original point cloud data

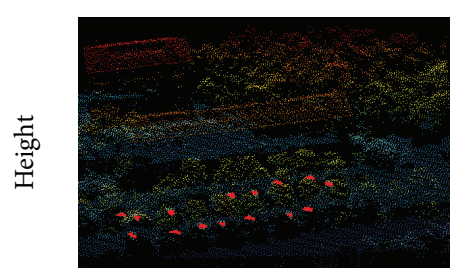

(c) Position of concealed cars

FIGURE 8: The CCD image, original point cloud data, and position of concealed target cars in experiment area II. 


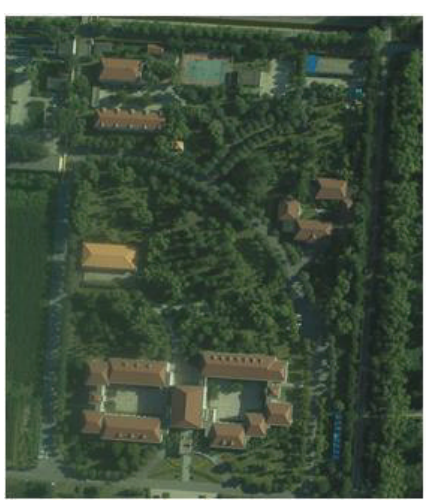

(a) CCD image

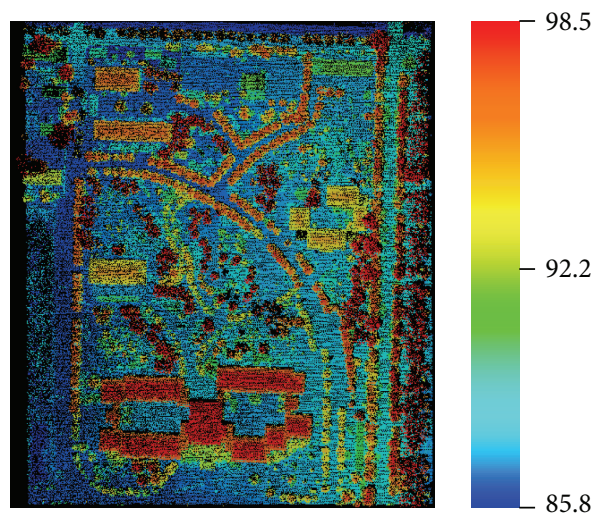

(b) Original point cloud data

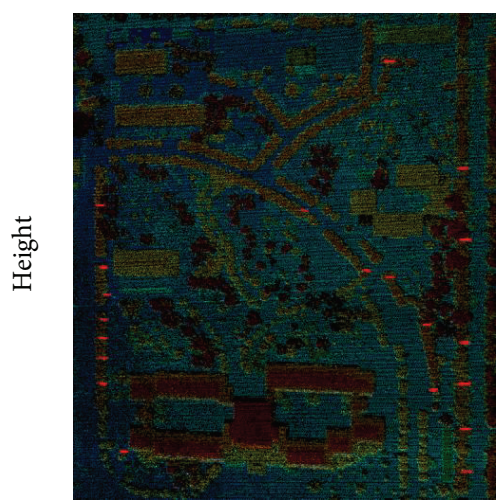

(c) Position of concealed cars

FIGURE 9: The CCD image, original point cloud data, and position of concealed target cars in experiment area III.

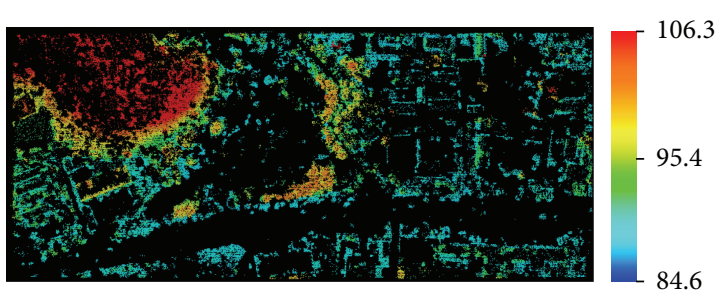

(a) Results of concealed cars areas using $G$ coefficient

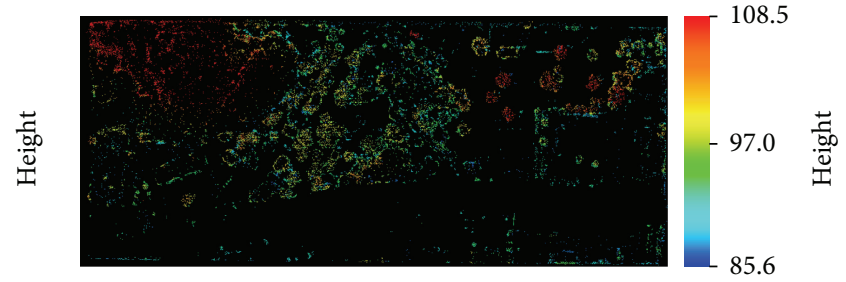

(b) Results of concealed cars areas using echo ratio

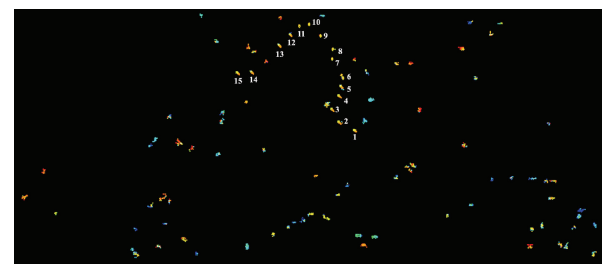

(c) Points segmentation results in concealed cars areas

FIGURE 10: Segmentation results of experiment area I.

segmented, which were marked by numbers in Figures 10(c), 11(c), and 12(c).

In order to verify the effectiveness of waveform features on concealed cars classification, comparative classification experiments were performed on the potential concealed cars shown in Figures 10(c), 11(c), and 12(c). First the classification experiment was conducted using waveform features and $\mathrm{VFH}$ descriptors. The other experiment was carried out with only the VFH descriptor.

The classification results using waveform features (intensity, waveform width, and backscattering cross section) and VFH descriptors for experiment area I, experiment area II, and experiment area III are, respectively, shown in Figures 13(a), 14(a), and 15(a). The classified cars numbered 2, 3, 4, 5, $6,7,8,10,11,12,13$, and 14 in Figure 13(a), numbered 1, 2, 4, $5,6,7,8,10,11,12$, and 14 in Figure 14(a), and numbered 1, 2, $3,4,5,6,7,9,10,11,12,13,15,16,18,19$, and 20 in Figure 15(a) are the true concealed cars; the remaining classified cars are the wrong targets. The classification results with only VFH descriptors are, respectively, shown in Figures 13(b), 14(b),
TABLE 1: Confusion matrix of binary classification problem.

\begin{tabular}{lcc}
\hline \multirow{2}{*}{ Confusion matrix } & \multicolumn{2}{c}{ Classified types } \\
& Concealed cars & Other objects \\
\hline Real types & TP & FN \\
Concealed cars & FP & TN \\
Other objects & FP
\end{tabular}

and 15(b). The classified cars numbered 1, 2, 3, 4, 5, 6, 8, 11, 12, and 15 in Figure 13(b), numbered 1, 2, 4, 5, 6, 8, 11, 12, and 15 in Figure 14(b), and numbered 1, 6, 10, 11, 12, 14, 15, 16, 18, 19 , and 20 in Figure 15(b) are the true concealed cars, while other classified cars are the wrong targets.

The classification in this paper is a binary classification problem and the confusion matrix of binary classification problem is shown in Table 1. Suppose ALL is the total number of clusters obtained in segmentation step and $C$ is the number of true concealed cars in the clusters. TP (True Positive) is the number of concealed cars that are correctly classified as 


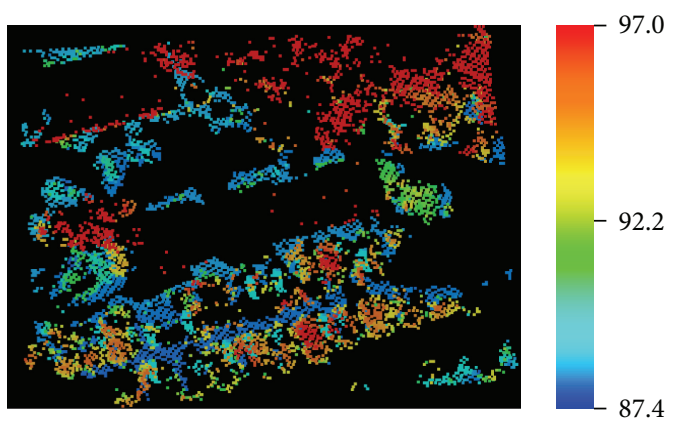

(a) Results of concealed cars areas using $G$ coefficient

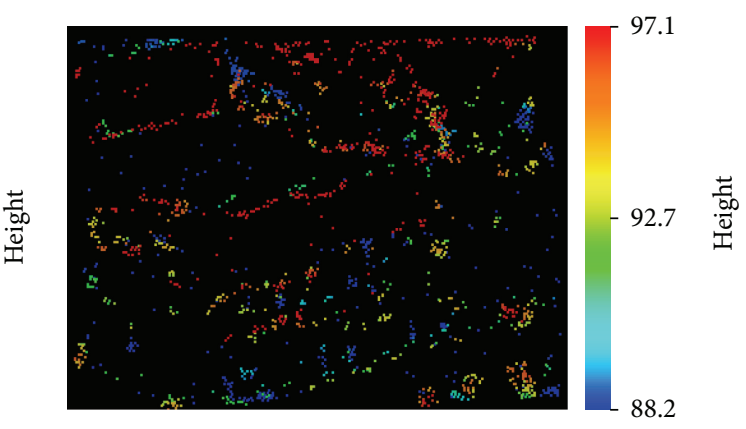

(b) Results of concealed cars areas using echo ratio

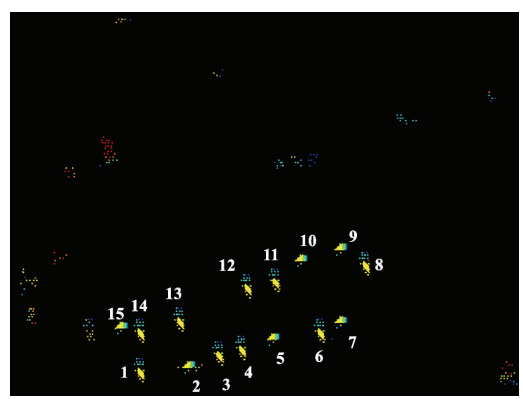

(c) Points segmentation results in concealed cars areas

FIgURE 11: Segmentation results of experiment area II.

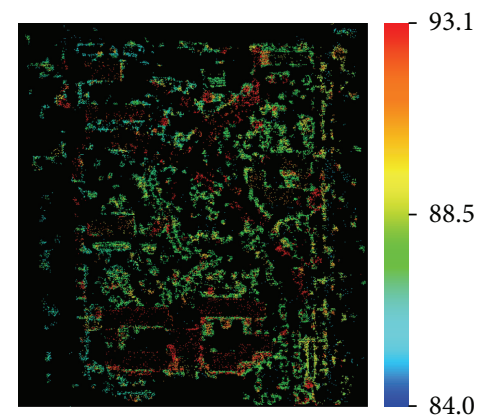

(a) Results of concealed cars areas using $G$ coefficient

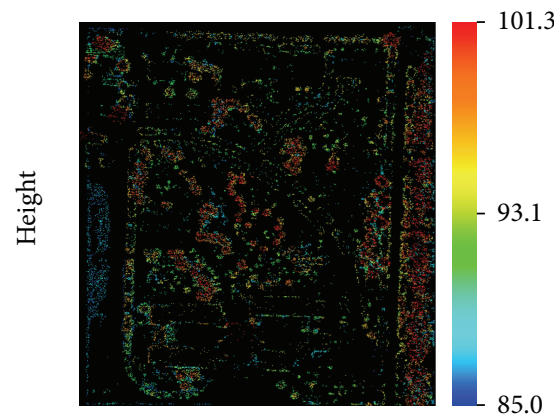

(b) Results of concealed cars areas using echo ratio

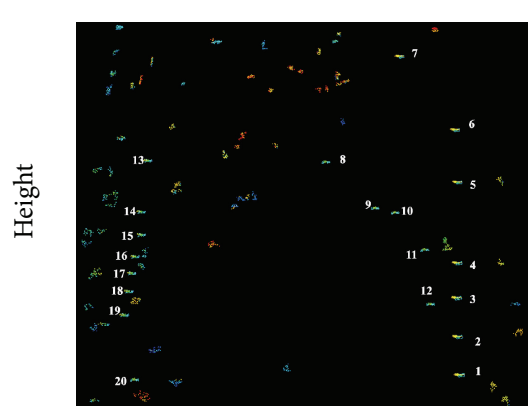

(c) Points segmentation results in concealed cars areas

FIGURE 12: Segmentation results of experiment area III.

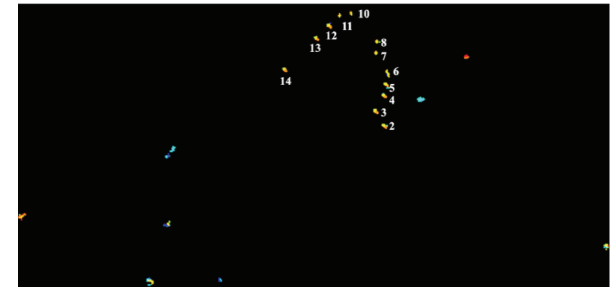

(a) Concealed car classification results with waveform features and VFH descriptors

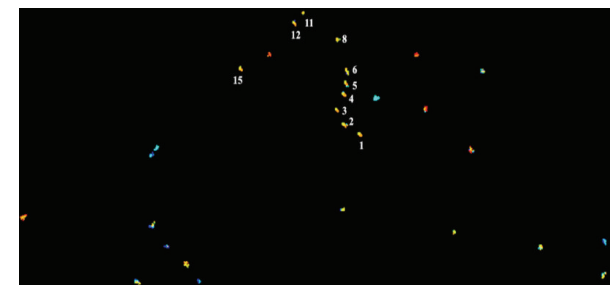

(b) Concealed car classification results with only VFH descriptors

FIGURE 13: Concealed car classification results in experiment area I. (a) Concealed car classification results with waveform features and VFH descriptors. (b) Concealed car classification results with only VFH descriptors. Each cluster in the figure was classified as a concealed car, and only the numbered cars were true concealed cars. 


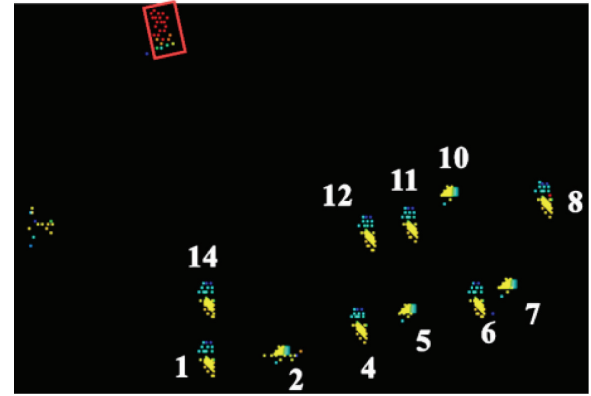

(a) Concealed car classification results with waveform features and VFH descriptors

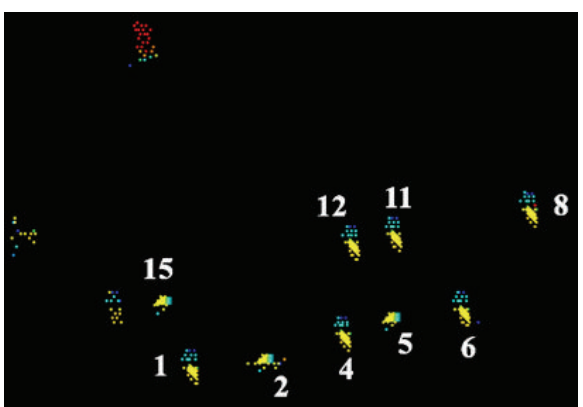

(b) Concealed car classification results with only VFH descriptors

FIGURE 14: Concealed car classification results in experiment area II. (a) Concealed car classification results with waveform features and VFH descriptors. (b) Concealed car classification results with only VFH descriptors. Each cluster in the figure was classified as a concealed car, and only the numbered cars were true concealed cars.

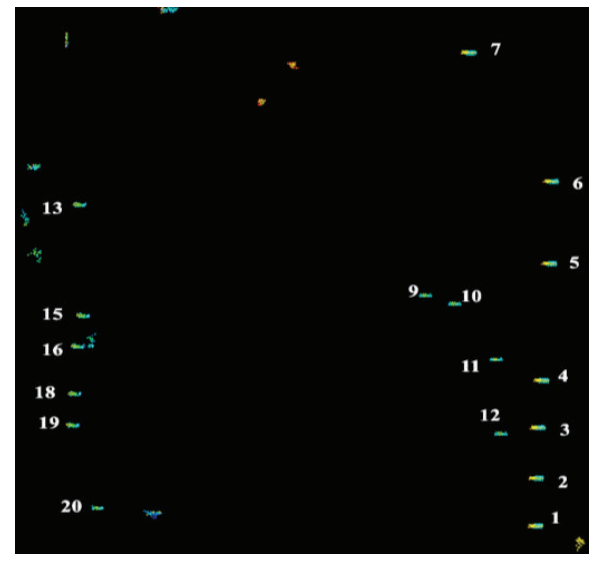

(a) Concealed car classification results with waveform features and VFH descriptors

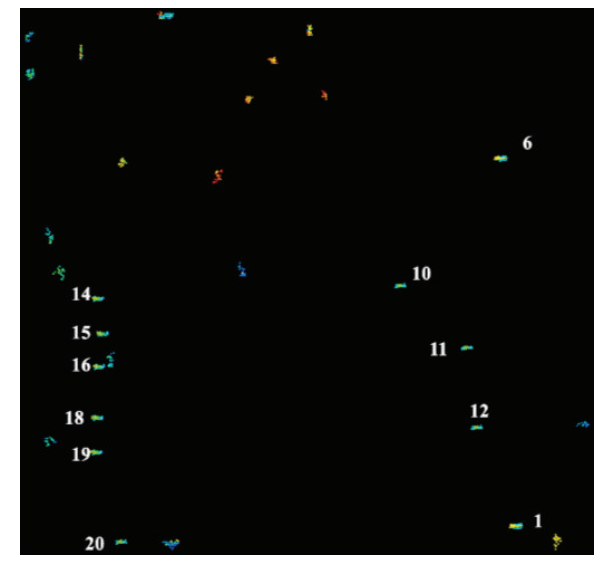

(b) Concealed car classification results with only VFH descriptors

FIGURE 15: Concealed car classification results in experiment area III. (a) Concealed car classification results with waveform features and VFH descriptors. (b) Concealed car classification results with only VFH descriptors. Each cluster in the figure was classified as a concealed car, and only the numbered cars were true concealed cars.

TABLE 2: Confusion matrix of the classification results with waveform features and VFH descriptors in experiment area I, area II, and area III (area I/area II/area III).

\begin{tabular}{lcc}
\hline \multirow{2}{*}{ Area I/area II/area III } & \multicolumn{2}{c}{ Classified types } \\
& Concealed cars & Other objects \\
\hline Real types & $12 / 11 / 17$ & $3 / 4 / 3$ \\
Concealed cars & $9 / 2 / 10$ & $72 / 11 / 52$ \\
Other objects & & \\
\hline
\end{tabular}

concealed cars. FP (False Positive) is the number of other objects (such as thickets beside the trees and bare cars beside the trees) that are falsely classified as concealed cars. TN (True Negative) is the number of other objects that are correctly classified as other objects. FN (False Negative) is the number of concealed cars that are falsely classified as other objects.
TABLE 3: Confusion matrix of the classification results with only VFH descriptors in experiment area I, area II, and area III (area I/area II/area III).

\begin{tabular}{lcc}
\hline \multirow{2}{*}{ Area I/area II/area III } & \multicolumn{2}{c}{ Classified types } \\
& Concealed cars & Other objects \\
\hline Real types & $10 / 9 / 11$ & \\
Concealed cars & $19 / 3 / 18$ & $6 / 6 / 9$ \\
Other objects & $62 / 10 / 44$ \\
\hline
\end{tabular}

The confusion matrix of the classification results with waveform features and VFH descriptors in experiment area I, area II, and area III is shown in Table 2.

The confusion matrix of the classification results with only VFH descriptors in experiment area I, area II, and area III is shown in Table 3. 
TABLE 4: Evaluation of concealed cars classification results.

\begin{tabular}{lcccc}
\hline \multicolumn{1}{c}{ Results } & & & \multicolumn{2}{c}{ Evaluation indices } \\
& & Recall & Precision & Accuracy \\
\hline \multirow{2}{*}{ Results with waveform features and VFH descriptors } & Area I & $80 \%(12 / 15)$ & $57.1 \%(12 / 21)$ & $87.5 \%(84 / 96)$ \\
& Area II & $73.3 \%(11 / 15)$ & $84.6 \%(11 / 13)$ & $78.6 \%(22 / 28)$ \\
& Area III & $85 \%(17 / 20)$ & $63.0 \%(17 / 27)$ & $84.1 \%(69 / 82)$ \\
\hline \multirow{3}{*}{ Results with only VFH descriptors } & Area I & $66.7 \%(10 / 15)$ & $34.5 \%(10 / 29)$ & $75 \%(72 / 96)$ \\
& Area II & $60 \%(9 / 15)$ & $75 \%(9 / 12)$ & $67.9 \%(19 / 28)$ \\
& Area III & $55 \%(11 / 20)$ & $37.9 \%(11 / 29)$ & $67.1 \%(55 / 82)$ \\
\hline
\end{tabular}

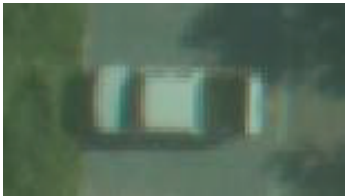

(a) CCD image

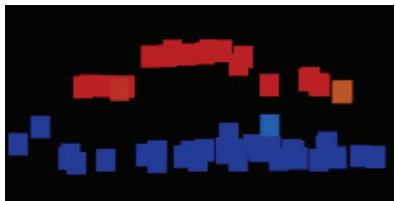

(b) Side view

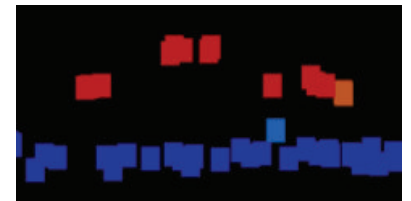

(c) Data thinning at the rate of $1 / 2$

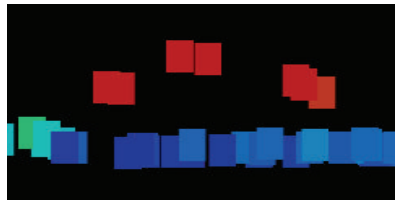

(d) Data thinning at the rate of $1 / 4$

FIGURE 16: CCD image and point cloud data before and after data thinning.

To evaluate the classification results, the indices including accuracy, recall, and precision [38] are most commonly used and defined as follows:

$$
\begin{aligned}
\text { Accuracy } & =\frac{\mathrm{TP}+\mathrm{TN}}{\mathrm{ALL}} \cdot 100 \%, \\
\text { Recall } & =\frac{\mathrm{TP}}{\mathrm{C}} \cdot 100 \%, \\
\text { Precision } & =\frac{\mathrm{TP}}{\mathrm{TP}+\mathrm{FP}} \cdot 100 \% .
\end{aligned}
$$

The evaluation results are shown in Table 4. By comparing the classification results with and without waveform features, we can see that, for the classification results using waveform features and VFH descriptors, the recall rate is improved by $13.3 \%, 13.3 \%$, and $30 \%$ and the precision and the accuracy are improved by $22.6 \%, 9.6 \%$, and $25.1 \%$ and $12.5 \%, 10.7 \%$, and $17 \%$ for these three experiment areas, respectively.

4.3. Results Analysis. Concluding from the experimented results, when the number of points of a concealed target is small, the random error of the features weakens the ability to distinguish different targets. This will greatly increase the difficulty of determining concealed targets. The point density of point cloud data, the target attributes, and the vegetation coverage factor may affect the number of points for concealed targets and then affect the concealed target extraction.

4.3.1. The Point Density of the Point Cloud Data. In order to analyze the influence of point density on concealed target extraction, different degrees of point cloud data thinning for concealed cars were applied. The data after data thinning was used for concealed cars extraction experiments. Figure 16 shows an example of data thinning results for a car. The point density of the experiment data was 4 points $/ \mathrm{m}^{2}$; thus the average distance between two points was $0.5 \mathrm{~m}$. There were approximately $20-30$ points obtained for each car in this case.
TABLE 5: Effect of point density on the number of cars' points.

\begin{tabular}{lccc}
\hline Point density (points $/ \mathrm{m}^{2}$ ) & 4 & 2 & 1 \\
\hline Number range of points for a car & $20-30$ & $10-15$ & $5-7$ \\
\hline
\end{tabular}

After thinning at the rate of $1 / 2$ or $1 / 4$, the point density of the experiment data decreased to 2 points $/ \mathrm{m}^{2}$ or 1 point $/ \mathrm{m}^{2}$. The number of points obtained for each car also decreased as shown in Table 5. Thus the density of point cloud data influences concealed cars extraction.

4.3.2. The Car Attributes. The size of a car and reflectivity affect the amount of point data that can be returned. Under the same condition, the bigger the target size, the more the points that can be obtained. With the decrease of the size of the target, the difficulty of target extraction increases gradually.

The target's reflectivity also affects the number of points. The numbers of points for the cars with different colors were analyzed by statistics, as shown in Table 6 . The greater the reflectivity, the more the points of the target that can be obtained. The reflectivity of the white car is greater than that of the black car, and the number of points from white car is bigger than that of black car. Thus the car attributes influence concealed cars extraction.

4.3.3. Vegetation Coverage Factor. In order to analyze the vegetation cover effect on concealed cars extraction, the vegetation coverage factor was calculated for some concealed cars as follows:

$$
\text { Vegetation coverage factor }=\frac{\text { Vegetation points }}{\text { Total points }} .
$$

From the results, as shown in Table 7, we can see that the number of points is bigger when vegetation coverage factor is smaller. It can be drawn that with the same targets' attributes 
TABLE 6: Effect of car attributes on the number of cars' points.

\begin{tabular}{lcccccccccccc}
\hline Car number & 1 & 2 & 3 & 4 & 5 & 6 & 7 & 8 & 9 & 10 \\
\hline Color & White & White & White & White & White & Red & Red & Red & Black & Black & Black \\
Number of points & 45 & 34 & 31 & 27 & 19 & 17 & 16 & 15 & 4 & 3 & 3 \\
\hline
\end{tabular}

TABLE 7: Vegetation coverage factor of concealed cars.

\begin{tabular}{lccccc}
\hline Car number & 1 & 2 & 3 & 4 & 5 \\
\hline $\begin{array}{l}\text { Vegetation } \\
\text { coverage }\end{array}$ & 0.44 & 0.8 & 0.65 & 0.59 & 0.67 \\
$\begin{array}{l}\text { factor } \\
\begin{array}{l}\text { Number of } \\
\text { points }\end{array}\end{array}$ & 4 & 4 & 11 & 34 & 10 \\
\hline
\end{tabular}

and point density, the smaller the vegetation cover factor is, the bigger the number of targets' points is and the more likely it is to extract the concealed targets. Thus the vegetation cover factor also influences concealed cars extraction.

Considering these factors as mentioned above, the errors of the concealed cars extraction were analyzed. The errors mainly came from two aspects. One issue was that some concealed cars were missed. That might be because these concealed cars were black or the vegetation cover factor was big for these cars; thus the number of points was not enough. It was difficult to extract the concealed cars with only a few points. The other issue was that some objects were wrongly classified as concealed cars, such as the dense thickets beside the trees or the bare car next to the trees. If the dense thickets beside the trees happened to have the similar elevation and waveform features with concealed cars then they might be classified as concealed cars. If the bare cars were very close to the trees and had enough points, then they might be classified as concealed cars, as shown in Figures 8(a) and 14(a) with the red rectangles.

In the future, the density of point cloud in the experiment area can be increased so that more points of the concealed cars can be obtained. Then the probability of missing the concealed cars can be decreased. Furthermore, more waveform features and geometry features should be extracted for better distinguish between dense thickets and concealed cars. In addition, more features can be used to accurately extract the points in vegetation areas so that the bare cars can be removed.

\section{Conclusion}

A concealed cars extraction algorithm was presented in this study. First $G$ coefficient and echo ratio were combined to determine concealed cars area based on full-waveform LiDAR data. The elevation distribution of the points in the concealed cars area was further analyzed to extract the concealed cars. Then by sample training and pattern matching, the concealed cars classification approach combining waveform features and VFH descriptor was presented. Experiments showed that the algorithm could correctly extract concealed cars, and comparative experiments further indicated that the waveform features could improve the accuracy, recall, and precision for concealed cars classification. However, this study focuses on concealed cars under trees; whether the algorithm is feasible to other types of concealed targets needs to be further investigated.

\section{Competing Interests}

The authors declare that there is no conflict of interests regarding the publication of this paper.

\section{Acknowledgments}

This work was partially supported by National Natural Science Foundation of China (Grant no. 40901177), National High Technology Research and Development Program of China (863 Program) (Grant no. 2015AA123801), National International Science and Technology Cooperation Project (Grant no. 2015DFA70930), and Key Projects of Foreign Cooperation in the International Cooperation Bureau of Chinese Academy of Sciences (Grant no. 181811KYSB20130003).

\section{References}

[1] K. Lai and D. Fox, "Object recognition in 3D point clouds using web data and domain adaptation," International Journal of Robotics Research, vol. 29, no. 8, pp. 1019-1037, 2010.

[2] B. C. Zhang, W. Smith, and S. Walker, "3-D object recognition from point clouds," http://velodynelidar.com/index.html.

[3] G. Vosselman and H.-G. Maas, Airborne and Terrestrial Laser Scanning, Whittles, 2010.

[4] D. C. Li, Bare-earth extraction and vehicle detection in forested terrain from airborne lidar point clouds [Ph.D. thesis], University of Florida, Gainesville, Fla, USA, 2010.

[5] W. Yao, S. Hinz, and U. Stilla, "Automatic vehicle extraction from airborne LiDAR data of urban areas aided by geodesic morphology," Pattern Recognition Letters, vol. 31, no. 10, pp. 1100-1108, 2010.

[6] M. L. Sun, L. I. Yong-Shu, Q. Chen et al., Automatic Car Extraction from Urban Airborne LiDAR Data Based on Height and Intensity Analysis, Remote Sensing Information, 2014.

[7] A. Borcs and C. Benedek, "Extraction of vehicle groups in airborne lidar point clouds with two-level point processes," IEEE Transactions on Geoscience and Remote Sensing, vol. 53, no. 3, pp. 1475-1489, 2015.

[8] L. D. Chang, K. C. Slatton, V. Anand et al., "Automatic forest canopy removal algorithm for underneath obscure target detection by airborne lidar point cloud data," in Proceedings of the Detection \& Sensing of Mines Explosive Objects \& Obscured Targets XV, vol. 7664 of Proceedings of SPIE, 12 pages, Orlando, Fla, USA, April 2010.

[9] L. A. Magruder and A. L. Neuenschwander, "Lidar fullwaveform data analysis for detection of faint returns through 
obscurants," in Proceedings of the Laser Radar Technology and Applications XIV, vol. 7323 of Proceedings of SPIE, Orlando, Fla, USA, May 2009.

[10] W. Wagner, A. Ullrich, V. Ducic, T. Melzer, and N. Studnicka, "Gaussian decomposition and calibration of a novel smallfootprint full-waveform digitising airborne laser scanner," ISPRS Journal of Photogrammetry and Remote Sensing, vol. 60, no. 2, pp. 100-112, 2006.

[11] C. Mallet and F. Bretar, "Full-waveform topographic lidar: stateof-the-art," ISPRS Journal of Photogrammetry \& Remote Sensing, vol. 64, no. 1, pp. 1-16, 2009.

[12] C. Alexander, K. Tansey, N. J. Tate et al., "Extraction of vegetation for topographic mapping from full-waveform airborne laser scanning data," in Proceedings of the Silvilaser 2008: The 8th International Conference on Lidar Applications in Forest Assessment and Inventory, pp. 343-353, Edinburgh, UK, September 2008.

[13] C. Mallet, F. Bretar, M. Roux, U. Soergel, and C. Heipke, "Relevance assessment of full-waveform lidar data for urban area classification," ISPRS Journal of Photogrammetry and Remote Sensing, vol. 66, no. 6, pp. S71-S84, 2011.

[14] N. Chehata, G. Li, and C. Mallet, "Airborne lidar feature selection for urban classification using random forests," Geomatics \& Information Science, vol. 38, pp. 207-212, 2009.

[15] M. Zhao, C. Zhao, and C. Zheng, "Identifying concealed information using wavelet feature extraction and support vector machine," Procedia Environmental Sciences, vol. 8, pp. 337-343, 2011.

[16] R. B. Rusu, G. Bradski, R. Thibaux, and J. Hsu, "Fast 3D recognition and pose using the viewpoint feature histogram," in Proceedings of the 23rd IEEE/RSJ International Conference on Intelligent Robots and Systems (IROS '10), pp. 2155-2162, IEEE, Taipei, Taiwan, October 2010.

[17] K. Alhamzi, M. Elmogy, and S. Barakat, "3D object recognition based on local and global features using point cloud library," International Journal of Advancements in Computing Technology, vol. 7, no. 3, pp. 43-54, 2015.

[18] A. Ceron and F. Prieto, "Evaluating and comparing of 3D shape descriptors for object recognition," in Advances in Visual Computing: 9th International Symposium, ISVC 2013, Rethymnon, Crete, Greece, July 29-31, 2013. Proceedings, Part II, vol. 8034 of Lecture Notes in Computer Science, pp. 484-492, Springer, Berlin, Germany, 2013.

[19] A. Aldoma, M. Vincze, N. Blodow et al., "CAD-model recognition and 6DOF pose estimation using 3D cues," in Proceedings of the IEEE International Conference on Computer Vision Workshops (ICCV '11), pp. 585-592, Barcelona, Spain, November 2011.

[20] S. Jha and P. Trivedi, "An automated video surveillance system using Viewpoint Feature Histogram and CUDA-enabled GPUs," in Proceedings of the 2nd International Conference on Advances in Computing, Communications and Informatics (ICACCI '13), pp. 1812-1816, Mysore, India, August 2013.

[21] Y. Salih, A. S. Malik, D. Sidibe et al., "Compressed VFH descriptor for 3D object classification," in Proceedings of the IEEE Transmission and Display of $3 D$ Video (3DTV-CON) 3DTV-Conference: The True Vision-Capture, pp. 1-4, Budapest, Hungary, July 2014.

[22] L. Guo, N. Chehata, C. Mallet, and S. Boukir, "Relevance of airborne lidar and multispectral image data for urban scene classification using Random Forests," ISPRS Journal of Photogrammetry and Remote Sensing, vol. 66, no. 1, pp. 56-66, 2011.
[23] M. Hussain and K. Fuchs, "Cluster analysis using spatial autocorrelation," in Data Analysis and Information Systems, pp. 52-63, Springer, Berlin, Germany, 1996.

[24] C. Fan and S. Myint, "A comparison of spatial autocorrelation indices and landscape metrics in measuring urban landscape fragmentation," Landscape \& Urban Planning, vol. 121, pp. 117128, 2014.

[25] L. Anselin, "Local indicators of spatial association-LISA," Geographical Analysis, vol. 27, no. 2, pp. 93-115, 1995.

[26] A. Getis and J. K. Ord, "The analysis of spatial association by use of distance statistics," Geographical Analysis, vol. 24, no. 3, pp. 189-206, 1992.

[27] H. Li, C. A. Calder, and N. Cressie, “Beyond Moran's I: testing for spatial dependence based on the spatial autoregressive model," Geographical Analysis, vol. 39, no. 4, pp. 357-375, 2007.

[28] R. B. Rusu, Z. C. Marton, N. Blodow, and M. Beetz, "Learning informative point classes for the acquisition of object model maps," in Proceedings of the 10th International Conference on Control, Automation, Robotics and Vision (ICARCV '08), pp. 643-650, IEEE, Hanoi, Vietnam, December 2008.

[29] R. B. Rusu, Semantic 3D object maps for everyday manipulation in human living environments [Ph.D. thesis], 2009.

[30] R. B. Rusu, N. Blodow, and M. Beetz, "Fast Point Feature Histograms (FPFH) for 3D registration," in Proceedings of the IEEE International Conference on Robotics and Automation (ICRA '09), pp. 3212-3217, IEEE Press, Kobe, Japan, May 2009.

[31] W. Wagner, J. Hyyppä, A. Ullrich et al., "Radiometric calibration of full-waveform small-footprint airborne laser scanners," International Archives of Photogrammetry, Remote Sensing and Spatial Information Sciences, vol. 37, part 1, pp. 163-168, 2008.

[32] W. Wagner, "Radiometric calibration of small-footprint fullwaveform airborne laser scanner measurements: Basic physical concepts," ISPRS Journal of Photogrammetry and Remote Sensing, vol. 65, no. 6, pp. 505-513, 2010.

[33] B. Höfle and M. Hollaus, "Urban vegetation detection using high density full-waveform airborne LIDAR datacombination of object-based image and point cloud analysis," The International Archives of the Photogrammetry Remote Sensing \& Spatial Information Sciences, vol. 38, pp. 281-286, 2010.

[34] H. Lehnera and C. Briesea, "Radiometric calibration of fullwaveform airborne laser scanning data based on natural surfaces," Years Isprs Advancing Remote Sensingence Pt, vol. 38, no. 1, pp. 360-365, 2010.

[35] M. Zhou, M. Liu, Z. Zhang et al., "The research of land covers classification based on waveform features correction of fullwaveform LiDAR," in Proceedings of the Image and Signal Processing for Remote Sensing XXI, vol. 9643 of Proceedings of SPIE, Toulouse, France, September 2015.

[36] M. Wang and Y.-H. Tseng, "Automatic segmentation of Lidar data into coplanar point clusters using an octree-based splitand-merge algorithm," Photogrammetric Engineering \& Remote Sensing, vol. 76, no. 4, pp. 407-420, 2010.

[37] R. Sinha, S. Samaddar, D. Bhattacharyya et al., "A tutorial on spatial data handling," International Journal of Database Theory \& Application, vol. 3, 2014.

[38] T. Fawcett, "An introduction to ROC analysis," Pattern Recognition Letters, vol. 27, no. 8, pp. 861-874, 2006. 

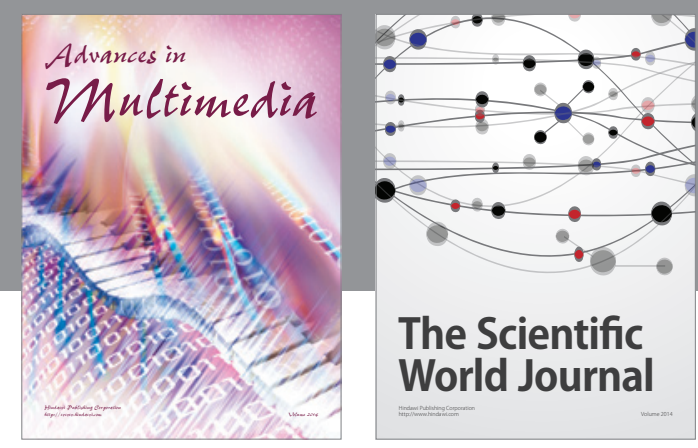

The Scientific World Journal
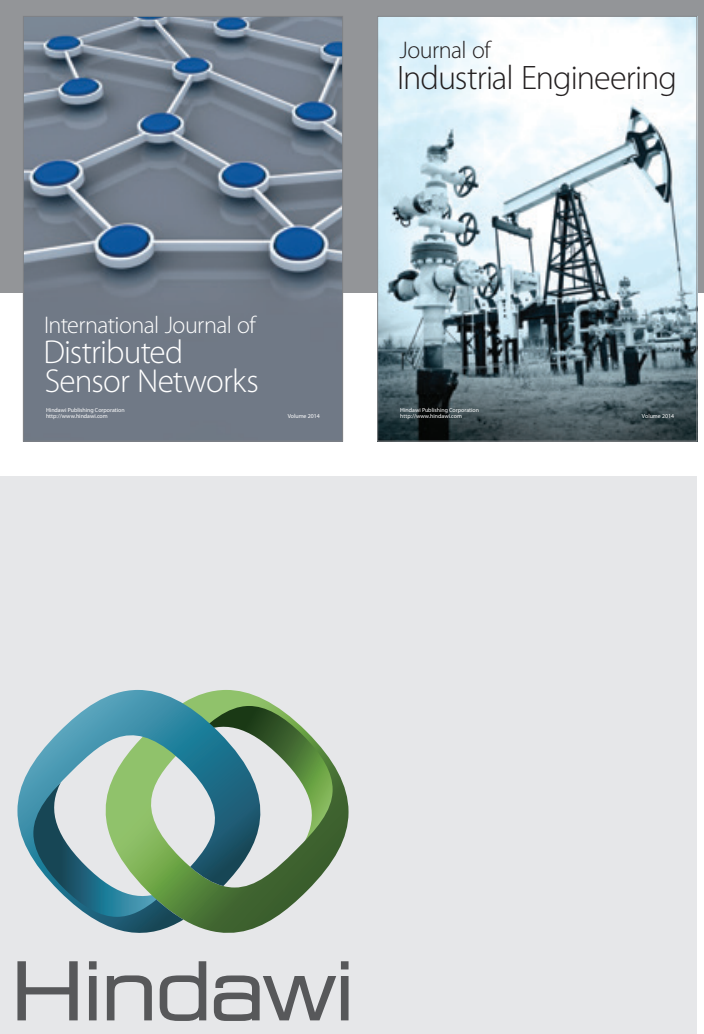

Submit your manuscripts at

http://www.hindawi.com

\section{Computer Networks} and Communications
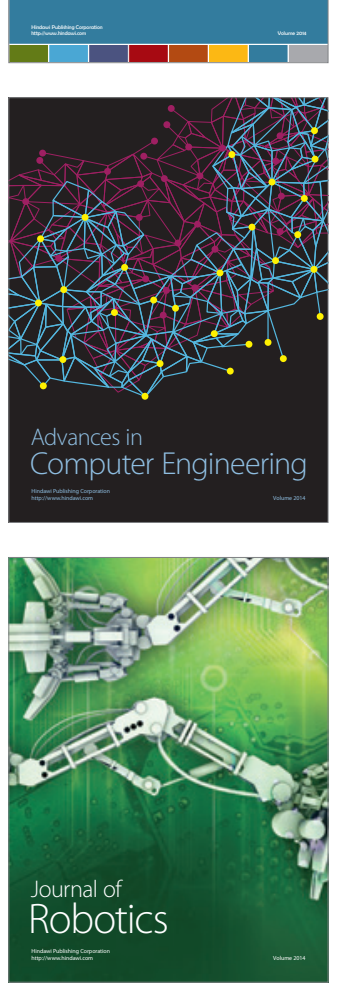
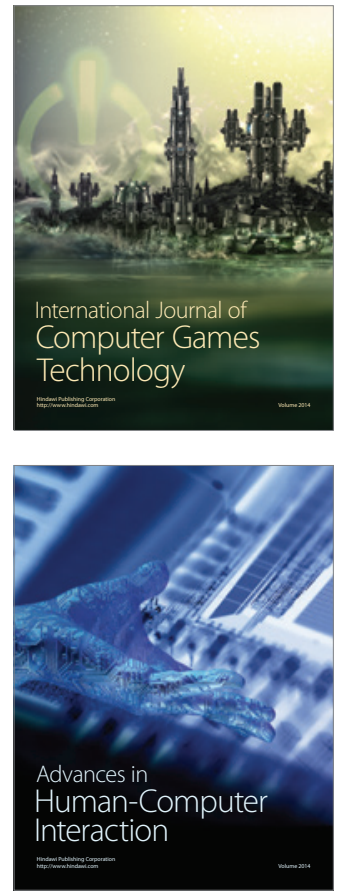
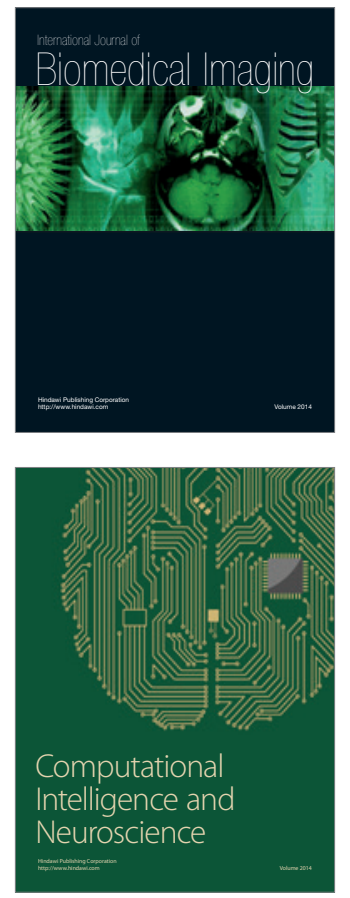
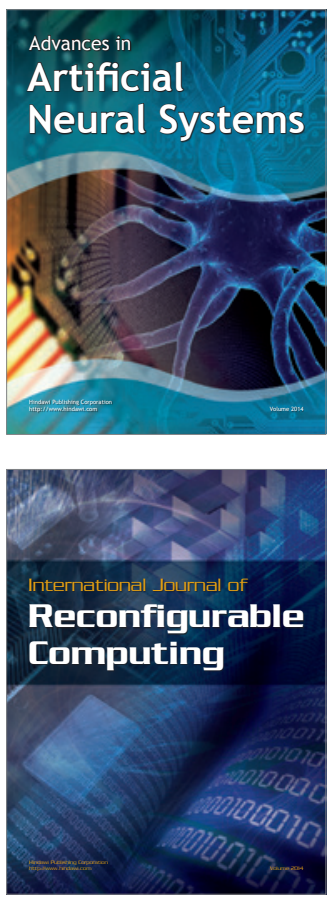
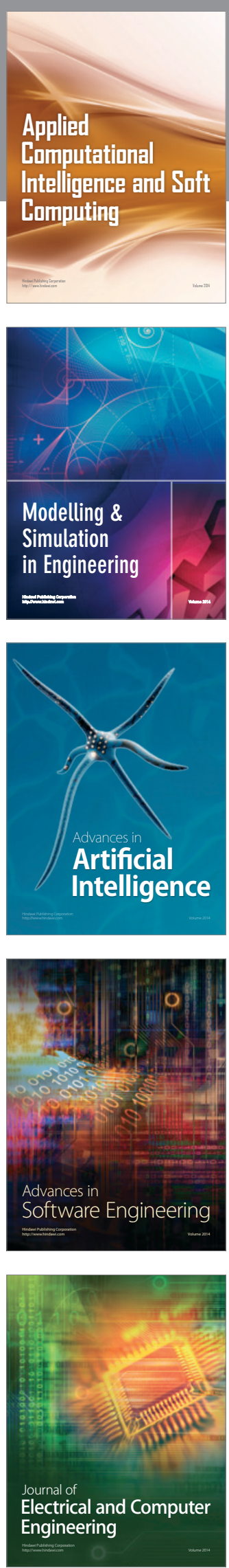